\section{New law risks release of invasive species}

A new European Union (EU) regulation aiming to control invasive alien species comes into force on 1 January 2015 (see go.nature.com/ajiwtd). It could inadvertently promote - rather than deter - the release of exotic animals into the wild.

The regulation prohibits the keeping, breeding, sale, movement and release of listed invasive alien species. The EU has set September 2015 as the deadline for the preliminary list: after this, member states will be required to consider eradication of listed species from within national borders.

Many species that are now among the worst invaders in Europe originated from private collections, which are at present governed only by piecemeal legislation. It is likely that several animals, which include the North American bullfrog (Lithobates catesbeianus), the ruddy duck (Oxyura jamaicensis), northern raccoon (Procyon lotor) and Pallas's squirrel (Callosciurus erythraeus), will be added to the list under the new ruling.

There is a risk, therefore, that this could prompt owners to release their newly illegal holdings into the wild, as happened after the 1997 EU trade ban on red-eared slider turtles (Trachemys scripta elegans).

A carefully managed interim process to prevent deliberate releases by pet owners should therefore be an EU-wide priority. Philip E. Hulme Lincoln University, Canterbury, New Zealand. philip.hulme@lincoln.ac.nz

\section{Harmful soot spurs climate-policy action}

Julia Schmale and colleagues rightly call for more policy action on short-lived climate-forcing pollutants (SLCPs) leading up to December's Conference of the Parties in Paris (Nature 515,
335-337; 2014). In fact, concerted action is already under way.

An alliance of countries, the Climate and Clean Air Coalition (www.ccacoalition.org), has made SLCPs a top priority. In November 2014, parties to the Montreal Protocol agreed to negotiate the phasing down of hydrofluorocarbons, one of the most potent SLCPs.

The substantial benefits of cutting these pollutants are now being recognized on many different fronts and in local and international arenas. Soot and other SLCPs kill millions of people and harm crops. Even countries that were once reluctant to adopt costly policies to mitigate climate change now find the political logic for action on SLCPs compelling.

Schmale et al. see tackling SLCPs as requiring new layers of international coordination. We see it differently: as a big opportunity to revitalize climatechange diplomacy, because action against SLCPs is seen by so many countries as being in their own interest.

David G. Victor, V. Ramanathan University of California San Diego, California, USA.

Durwood Zaelke Institute for Governance and Sustainable Development, Washington DC, USA.

david.victor@ucsd.edu

\section{Green heating plan threatens air quality}

The UK government's

Renewable Heat Incentive (RHI)

aims to transform domestic and non-domestic heating with affordable biomass-only boilers and pellet stoves. This could have unwanted consequences for air quality and climate change unless the RHI rapidly reduces its emissions limits (see also J. Schmale et al. Nature 515, 335-337; 2014).

The RHI stipulates an airquality limit for biofuel burning of 30 grams of particulate matter per gigajoule of net heat output.
This means that a 10-kilowatt unit operating for 2.8 hours is allowed to emit as much as $3 \mathrm{~g}$ of particulates of up to 10 micrometres in size $\left(\mathrm{PM}_{10}\right)$ in a total of 27 cubic metres of boiler exhaust fumes (111 milligrams per cubic metre). Under current UK air-quality regulations, the permitted ambient $\mathrm{PM}_{10}$ level is about 2,000 times lower than this; the European $\mathrm{PM}_{10}$ limit is 5,000 times lower.

The RHI impact assessment suggests supporting 750,000 systems by 2020 . Yet, if just $2.5 \%$ of the UK homes now heated by natural gas ( 500,000 boilers) were to switch to RHI-compliant 10-kW biomass boilers (enough to power five double radiators) and use them for 2.8 hours, this would generate the same mass of particulates as would be emitted in one day by the entire UK lightduty diesel fleet of 8.8 million vehicles were they to operate under the latest Euro 5 emissions regulations (see go.nature.com/ mjpqmz).

Furthermore, much of the particulate matter emitted by biomass boilers is sooty black carbon - a climate-warming agent owing to its absorption of outgoing longwave radiation. So the intended positive effect of the RHI on climate will be compromised.

Gordon McFiggans University of Manchester, UK. g.mcfiggans@manchester.ac.uk

\section{Criteria for Nature Index questioned}

The Nature Index gauges the performance of countries and research institutions by tracking the number of papers they publish in reputable journals (www.natureindex.com). We suggest that using absolute numbers and journal reputation can yield misleading results (see R. Haunschild and L. Bornmann Scientometrics (in the press) and at http://doi.org/xrg; 2014).

For example, the index ranks the Chinese Academy of Sciences above Harvard University in Cambridge, Massachusetts. Using the complete publication output from each of these in 2013 (31,428 and 17,836 articles, respectively, according to Thomson Reuters InCites), we calculate that only $8 \%(2,661)$ of papers from the Chinese Academy of Sciences contributed to the Nature Index, whereas $14 \%$ (2,555 papers) of Harvard papers contributed. This relative perspective is important, given that an institution with a high publication output would be expected to publish more papers in reputable journals.

In our view, it would also be better to measure the performance of countries and institutions on the basis of individual papers, rather than on the journals in which they are published (see http://am.ascb. org/dora). This is because the quality of a journal (as measured by peers or citations) is not a reliable proxy for the quality of each paper it publishes.

To illustrate this point, we counted citations over 5 years for papers published in 2008 in Applied Physics Letters - the journal contributing most articles to the Nature Index using an in-house bibliometrics database maintained by the Max Planck Digital Library in Munich, Germany. We found that some $40 \%$ of the papers in Applied Physics Letters accounted for about $80 \%$ of the citations to this journal, suggesting an uneven distribution of quality.

Robin Haunschild Max Planck Institute for Solid State Research, Stuttgart, Germany.

Lutz Bornmann Max Planck Society, Munich, Germany. r.haunschild@fkf.mpg.de

Editorial note: For details of Nature Index methodology, see Nature 515, S52-S53 (2014). A response from Nature Publishing Group to R. Haunschild and L. Bornmann's forthcoming Scientometrics article is available at http://doi.org/xsf (2014). 